\title{
Field in Focus with a Maximum Longitudinal Electric Component
}

\author{
H.P. Urbach ${ }^{1,2, *}$ and S.F. Pereira ${ }^{1}$ \\ ${ }^{1}$ Optics Research Group, Department of Imaging Science and Technology, Delft University of Technology, \\ P.O. Box 5046, 2600 GA Delft, The Netherlands \\ ${ }^{2}$ Philips Research Laboratories, High Tech Campus 36, 5656 AA Eindhoven, The Netherlands \\ (Received 30 October 2007; revised manuscript received 25 January 2008; published 27 March 2008)
}

Closed formulas are presented for the field in the lens pupil for which the longitudinal electric component at the focal point is larger than any other focused field with the same power. The fullwidth-at-half-maximum of the squared amplitude of the maximum longitudinal component is $15 \%$ to $30 \%$ less than that of the classical Airy spot.

DOI: 10.1103/PhysRevLett.100.123904

PACS numbers: 42.25.Hz, 42.25.Fx, 42.25.Ja

Introduction. - When a linearly polarized plane wave is focused by a diffraction-limited lens of small numerical aperture, the intensity distribution in the focal plane is the normal Airy pattern. When the lens has high numerical aperture, the rotation of polarization must be accounted for using the vectorial diffraction theory of Ignatowsky [1] (published already in 1919), and Richards and Wolf [2,3], and then the vectorial Airy pattern is obtained. The dominant field component in the focal plane of the vectorial Airy pattern is as expected parallel to the incident polarization. But for large numerical aperture the longitudinal component of the electric field in the focal plane becomes quite substantial, although it vanishes exactly at the focal point itself.

Radially polarized beams are locally linearly polarized, where the electric field in the entrance pupil is pointing in the radial direction with respect to the optical axis. When such a beam is focused, the longitudinal component attains its maximum at the focal point. Moreover, for the same numerical aperture, the distribution of the longitudinal component can be considerably narrower than the Airy pattern [4-7]. A small and appropriately shaped focused spot is essential in many applications such as optical recording, photolithography, and microscopy. New photoresists have already been proposed that bleach in response to only one field component [8]. Materials with molecules with fixed absorption dipole moments were applied in [9] to probe field components individually. When this component is the longitudinal component, a higher resolution can thus be obtained than with the classical Airy pattern. The narrow longitudinal component has also attracted a lot of interest in areas such as the manipulation of single molecules and particles, and material processing [9-12].

Often the amplitude distribution in the pupil plane of the radially polarized beam is taken to be a doughnut shape or a ring mask function [4,5]. But this does not give the largest possible longitudinal component. In this Letter we present closed formulas for the electric field in the lens pupil for which the longitudinal electric field component in the focal point is larger than for any other focused field with the same power. The optimum pupil field is found to be radially polarized; however, the amplitude is not of doughnut type but increases monotonically with distance to the optical axis in a way that depends on the numerical aperture. This pupil field is easy to realize using, for example, liquid crystal-based devices [13-16]. The full-width-athalf-maximum of the squared amplitude of the maximum longitudinal component is, depending on the numerical aperture, $15 \%$ to $30 \%$ less than that of the classical Airy spot.

We first determine the plane wave amplitudes of the optimum field in the focal region, without considering the lens. Then we derive the field in the pupil of the lens which, according to the vectorial diffraction theory of Ignatovsky and Richards and Wolf, yields the optimum field in the focal region. The advantage of this procedure is that the obtained optimum plane wave amplitudes are independent of the vectorial diffraction model of the lens. With respect to a coordinate system $(x, y, z)$, we consider a timeharmonic electromagnetic field $\mathcal{E}(\mathbf{r}, t)=\operatorname{Re}\left[\mathbf{E}(\mathbf{r}) e^{-i \omega t}\right]$, $\mathcal{H}(\mathbf{r}, t)=\operatorname{Re}\left[\mathbf{H}(\mathbf{r}) e^{-i \omega t}\right]$, (with $\omega>0$ ) in a homogeneous unbounded medium in $z>0$ with real refractive index $n$. The plane wave expansion of the field consists of propagating plane waves only, with numerical aperture $N A$ :

$$
\begin{aligned}
& \mathbf{E}(\mathbf{r})=\frac{1}{4 \pi^{2}} \iint_{\sqrt{k_{x}^{2}+k_{y}^{2}} \leq N A k_{0}} \mathbf{A}\left(k_{x}, k_{y}\right) e^{i \mathbf{k} \cdot \mathbf{r}} d k_{x} d k_{y}, \\
& \mathbf{H}(\mathbf{r})=\frac{1}{4 \pi^{2}} \frac{1}{\omega \mu_{0}} \iint_{\sqrt{k_{x}^{2}+k_{y}^{2}} \leq N A k_{0}} \mathbf{k} \\
& \times \mathbf{A}\left(k_{x}, k_{y}\right) e^{i \mathbf{k} \cdot \mathbf{r}} d k_{x} d k_{y},
\end{aligned}
$$

where $\mathbf{k}=\left(k_{x}, k_{y}, k_{z}\right)$ and $k_{0}=\omega \sqrt{\epsilon_{0} \mu_{0}}=2 \pi / \lambda_{0}$ the wave number in vacuum, and where $N A=n \sin \vartheta_{\max }$, with $\vartheta_{\max }$ the maximum angle between the wave vectors and the positive $z$ direction. Note that apart from the limitation on the numerical aperture, these plane wave expansions are completely arbitrary. We shall use spherical coordinates $k, \vartheta, \varphi$ in $\mathbf{k}$ space. The unit vectors of the (positively orientated) basis are 


$$
\begin{aligned}
& \hat{\mathbf{k}}=\sin \vartheta \cos \varphi \hat{\mathbf{x}}+\sin \vartheta \sin \varphi \hat{\mathbf{y}}+\cos \vartheta \hat{\mathbf{z}}, \\
& \hat{\boldsymbol{\vartheta}}=\cos \vartheta \cos \varphi \hat{\mathbf{x}}+\cos \vartheta \sin \varphi \hat{\mathbf{y}}-\sin \vartheta \hat{\mathbf{z}} \\
& \hat{\boldsymbol{\varphi}}=-\sin \varphi \hat{\mathbf{x}}+\cos \varphi \hat{\mathbf{y}}
\end{aligned}
$$

Since the electric field is free of divergence, the electric field of the plane waves have only $\vartheta$ and $\varphi$ components:

$$
\mathbf{A}(\varphi, \vartheta)=A_{\vartheta}(\varphi, \vartheta) \hat{\boldsymbol{\vartheta}}+A_{\varphi}(\varphi, \vartheta) \hat{\boldsymbol{\varphi}}
$$

The plane wave expansion (1) and (2) can then be written as integrals over $\vartheta$ and $\varphi$ :

$$
\begin{aligned}
& \mathbf{E}(\mathbf{r})=\frac{n^{2}}{\lambda_{0}^{2}} \int_{0}^{2 \pi} \int_{0}^{\vartheta_{\text {max }}}\left(A_{\vartheta} \hat{\boldsymbol{\vartheta}}+A_{\varphi} \hat{\boldsymbol{\varphi}}\right) \\
& \times \sin \vartheta \cos \vartheta e^{i \mathbf{k} \cdot \mathbf{r}} d \varphi d \vartheta \text {, } \\
& \mathbf{H}(\mathbf{r})=\frac{n^{3}}{\lambda_{0}^{2}}\left(\frac{\epsilon_{0}}{\mu_{0}}\right)^{1 / 2} \int_{0}^{2 \pi} \int_{0}^{\vartheta_{\max }}\left(-A_{\varphi} \hat{\boldsymbol{\vartheta}}+A_{\vartheta} \hat{\boldsymbol{\varphi}}\right) \\
& \times \sin \vartheta \cos \vartheta e^{i \mathbf{k} \cdot \mathbf{r}} d \varphi d \vartheta . \\
& E_{z}(\mathbf{0})=-\frac{n^{2}}{\lambda_{0}^{2}} \int_{0}^{2 \pi} \int_{0}^{\vartheta_{\max }} A_{\vartheta}(\varphi, \vartheta) \sin ^{2} \vartheta \cos \vartheta d \varphi d \vartheta .
\end{aligned}
$$

We may assume that $E_{z}(\mathbf{0})$ is real (if it is not real, apply a time shift to make it real). In the optimization problem, $E_{z}(\mathbf{0})$ is considered as a functional of $A_{\vartheta}$. By using Plancherel's formulas, the total time-averaged power flow in the $z$ direction through a plane $z=$ const can be expressed as an integral over contributions of plane waves, i.e., as an integral over $k_{x}$ and $k_{y}$. By changing to integration variables $\vartheta, \varphi$ we get

$$
\begin{aligned}
P= & \int_{-\infty}^{\infty} \int_{-\infty}^{\infty} \frac{1}{2} \operatorname{Re}\left[\mathbf{E}(\mathbf{r}) \times \mathbf{H}(\mathbf{r})^{*}\right] \cdot \hat{\mathbf{z}} d x d y \\
= & \frac{n^{3}}{2 \lambda_{0}^{2}}\left(\frac{\epsilon_{0}}{\mu_{0}}\right)^{1 / 2} \int_{0}^{2 \pi} \int_{0}^{\vartheta_{\max }}\left[\left|A_{\vartheta}(\varphi, \vartheta)\right|^{2}\right. \\
& \left.+\left|A_{\varphi}(\varphi, \vartheta)\right|^{2}\right] \cos ^{2} \vartheta \sin \vartheta d \varphi d \vartheta .
\end{aligned}
$$

We shall write $P\left(A_{\vartheta}, A_{\varphi}\right)$ for the power flow. It is independent of the plane $z=$ const, as should be in a medium without losses.

The optimization problem is to find the plane wave amplitudes $\mathbf{A}=A_{\vartheta} \hat{\boldsymbol{\vartheta}}+A_{\varphi} \hat{\boldsymbol{\varphi}}$ for which the amplitude of the longitudinal electric field at the origin is maximum among all fields with the same mean power flow $P_{0}$, i.e.,

$$
\text { maximize } E_{z}(\mathbf{0}) \text {, }
$$$$
\text { under the constraint } P\left(A_{\vartheta}, A_{\varphi}\right)=P_{0} \text {. }
$$

Since the object functional $E_{z}(\mathbf{0})$ depends linearly on the plane wave amplitudes and the energy constraint functional $P$ is quadratic in these amplitudes, the optimization problem is a classical quadratic problem. The Lagrange multiplier rule therefore yields a system of linear equations for the optimum plane wave amplitudes. By using this system of equations the optimum plane wave amplitudes can be expressed in the Lagrange multiplier $\Lambda$ and one finds

$$
A_{\vartheta}(\vartheta, \varphi)=-\frac{\tan \vartheta}{\Lambda}, \quad A_{\varphi}(\vartheta, \varphi)=0
$$

The Lagrange multiplier is obtained by substituting (8) into the constraint $P\left(A_{\vartheta}, A_{\varphi}\right)=P_{0}$. This gives

$$
\Lambda=\left(\frac{\pi}{P_{0}}\right)^{1 / 2} \frac{n^{3 / 2}}{\lambda_{0}}\left(\frac{\epsilon_{0}}{\mu_{0}}\right)^{1 / 4}\left(\frac{2}{3}-\cos \vartheta_{\max }+\frac{1}{3} \cos ^{3} \vartheta_{\max }\right)^{1 / 2} .
$$

The maximum value of $E_{z}(\mathbf{0})$ is

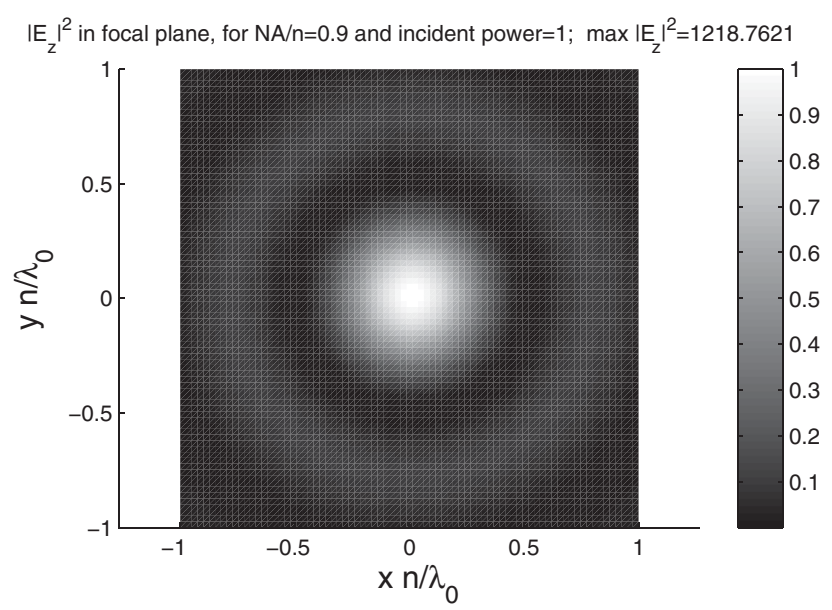

(a)

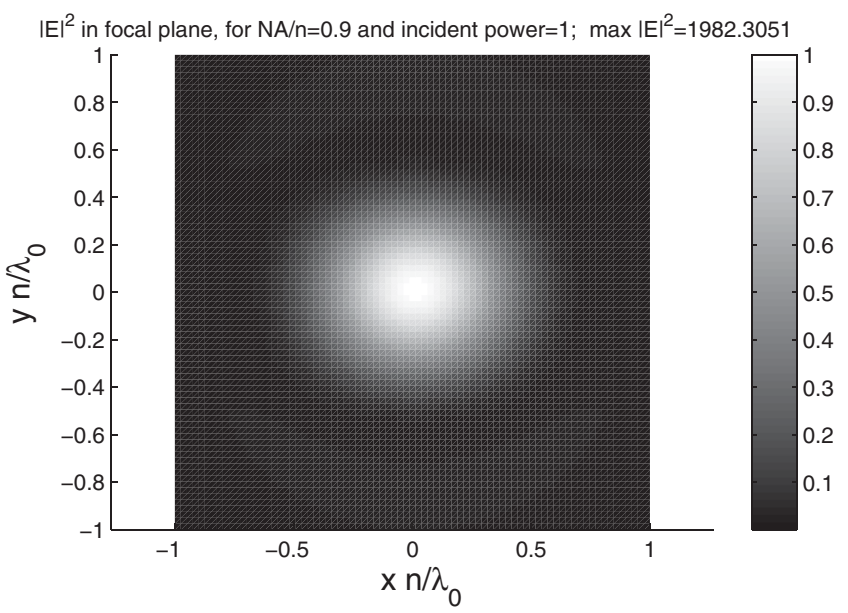

(b)

FIG. 1. (a) Normalized $\left|E_{z}\right|^{2}$ of the optimum field in the $z=0$ plane and (b) normalized electric energy distribution $|\mathbf{E}(\mathbf{r})|^{2}$ in the focal plane of an $x$-polarized focused plane wave. The total power flow is the same for both fields and $N A / n=0.9$. 


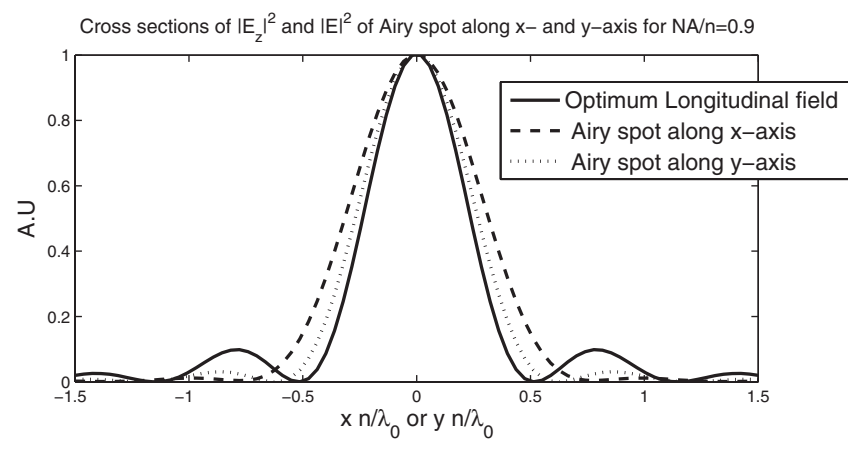

FIG. 2. Cross-sections of the optimum $\left|E_{z}(x, y, 0)\right|^{2}$ (solid curves) and of $|\mathbf{E}(x, 0,0)|^{2}$ (dashed) and $|\mathbf{E}(0, y, 0)|^{2}$ (dotted) for the Airy spot of the $x$-polarized focused plane wave, for $N A / n=0.9$.

$$
\begin{aligned}
E_{z}(\mathbf{0})_{\max } & =\frac{1}{\Lambda} \frac{n^{2}}{\lambda_{0}^{2}} \int_{0}^{2 \pi} \int_{0}^{\vartheta_{\max }} \sin ^{3} \vartheta d \varphi d \vartheta \\
& =\frac{2 \pi}{\Lambda} \frac{n^{2}}{\lambda_{0}^{2}}\left(\frac{2}{3}-\cos \vartheta_{\max }+\frac{1}{3} \cos ^{3} \vartheta_{\text {max }}\right)=2 P_{0} \Lambda
\end{aligned}
$$

By substituting (8) into (4) and (5) and computing the integrals over $\varphi$, we find for the optimum electromagnetic field in a point $\mathbf{r}$ with cylindrical coordinates $\varrho, \varphi, z$ :

$$
\begin{gathered}
\mathbf{E}(\mathbf{r})=-2 \pi i \frac{n^{2}}{\Lambda \lambda_{0}^{2}}\left[g_{1}^{1,2}(\varrho, z) \hat{\boldsymbol{\varrho}}+i g_{0}^{0,3}(\varrho, z) \hat{\mathbf{z}}\right], \\
\mathbf{H}(\mathbf{r})=-2 \pi i \frac{n^{3}}{\Lambda \lambda_{0}^{2}}\left(\frac{\epsilon_{0}}{\mu_{0}}\right)^{1 / 2} g_{1}^{0,2}(\varrho, z) \hat{\boldsymbol{\varphi}},
\end{gathered}
$$

where $\hat{\boldsymbol{\varrho}}, \hat{\boldsymbol{\varphi}}$, and $\hat{\mathbf{z}}$ are the unit vectors of the cylindrical coordinate system:

$$
\hat{\boldsymbol{\varrho}}=\cos \varphi \hat{\mathbf{x}}+\sin \varphi \hat{\mathbf{y}}, \quad \hat{\boldsymbol{\varphi}}=-\sin \varphi \hat{\mathbf{x}}+\cos \varphi \hat{\mathbf{y}},
$$

and where $g_{l}^{\nu, \mu}$ is defined by

$g_{l}^{\nu, \mu}(\varrho, z)=\int_{0}^{\vartheta_{\max }} e^{i k_{0} n z \cos \vartheta} \cos ^{\nu} \vartheta \sin ^{\mu} \vartheta J_{l}\left(k_{0} n \varrho \sin \vartheta\right) d \vartheta$.

The time-averaged Poynting vector is

$$
\begin{aligned}
\frac{1}{2} \operatorname{Re}\left[\mathbf{E}(\mathbf{r}) \times \mathbf{H}(\mathbf{r})^{*}\right]= & \frac{2 \pi^{2} n^{5}}{\tilde{\Lambda}^{2} \lambda_{0}^{4}}\left(\frac{\epsilon_{0}}{\mu_{0}}\right)^{1 / 2}\left\{\operatorname{Im}\left[g_{0}^{0,3}\left(g_{1}^{0,2}\right)^{*}\right] \hat{\boldsymbol{\varrho}}\right. \\
& \left.+\operatorname{Re}\left[g_{1}^{1,2}\left(g_{1}^{0,2}\right)^{*}\right] \hat{\mathbf{z}}\right\} .
\end{aligned}
$$

Since $g_{l}^{\nu, \mu}$ are independent of $\varphi$, so is $E_{z}$. Furthermore, for all points in the (focal) $z=0$ plane, the Poynting vector is parallel to the $z$ direction. In Fig. 1(a) the (normalized) $\left|E_{z}\right|^{2}$ is shown in the $z=0$ plane for the field with maximum longitudinal component at the origin. For comparison the normalized total electric energy density: $|\mathbf{E}(x, y, 0)|^{2}=$ $\left|E_{x}(x, y, 0)\right|^{2}+\left|E_{y}(x, y, 0)\right|^{2}+\left|E_{z}(x, y, 0)\right|^{2}$, in the focal

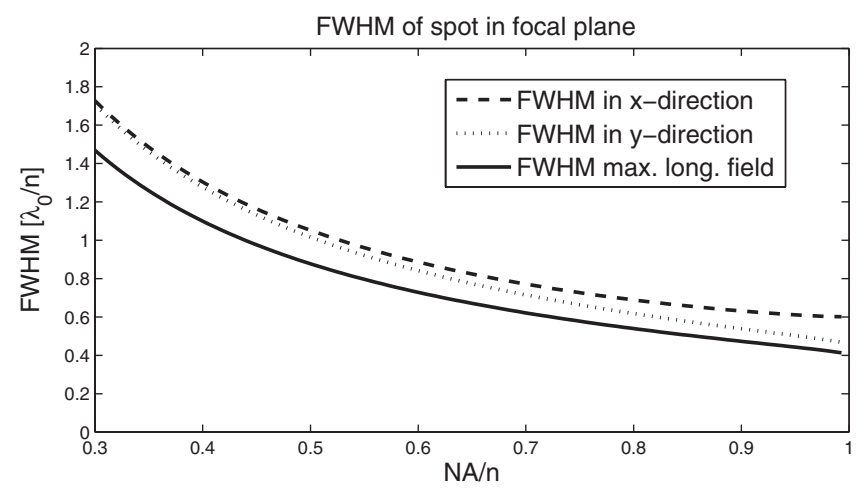

FIG. 3. FWHM of $\left|E_{z}(x, y, 0)\right|^{2}$ of the optimum field and FWHM in the $x$ and $y$ direction of the focused $x$-polarized plane wave as a function of $N A / n$.

$z=0$ plane of a focused $x$-polarized plane wave [1-3] is shown in Fig. 1(b). The fields have the same total power flow in the $z$ direction. In contrast to $\left|E_{z}(x, y, 0)\right|^{2}$, the energy density of the focused linear polarized plane wave has elliptical shape. Cross sections along the short and long axes of the ellipse are shown in Fig. 2 for $N A / n=0.9$. The longitudinal component has smaller FWHM but also stronger secondary maxima. In Fig. 3 the FWHM of the optimum $\left|E_{z}(x, y, 0)\right|^{2}$ is compared to the FWHM in the $x$ and $y$ directions of the electric energy density of the focused $x$-polarized plane wave. Depending on the value of $N A$, the FWHM of the optimum longitudinal component is $15 \%-30 \%$ smaller than that of the energy density of the focused plane wave. Interestingly, the FWHM of the optimum longitudinal component is for $N A / n=1$ almost identical to that of the longitudinal component in [5], obtained by focusing a radially polarized beam using a ring mask function (with radius $90 \%$ of the total pupil). However, the side lobes are higher at the cost of the central maximum compared to our longitudinal component. This is of course not surprising because the longitudinal component in [5] was not optimized for a high maximum on the optical axis.

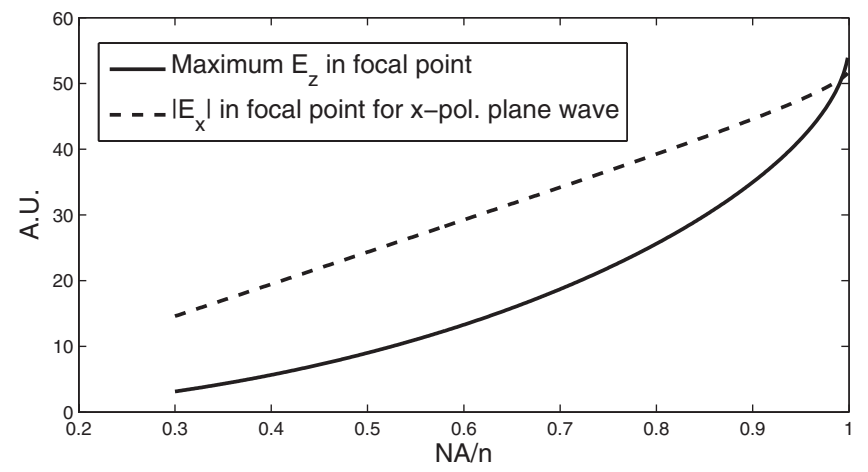

FIG. 4. Comparison of the maximum $E_{z}(\mathbf{0})$ and the maximum of $\left|E_{x}\right|$ in the focal point when a $x$-polarized plane wave is focused. The total flow of power is the same for both fields. 


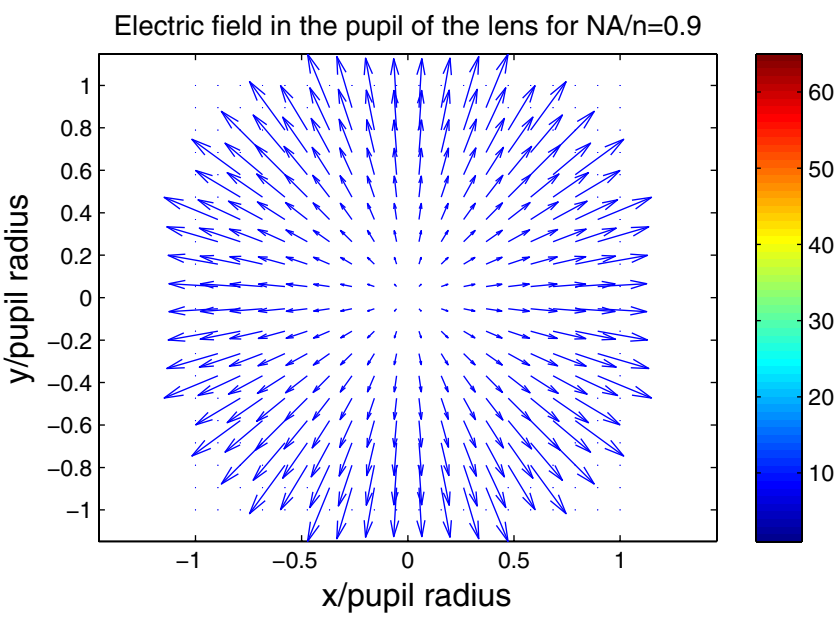

FIG. 5 (color online). The electric field in the pupil that, when focused, yields the field with maximum longitudinal component at the focal point for $N A / n=0.9$.

In Fig. 4 the maximum longitudinal component (10) as a function of $N A / n$ is compared with $\left|E_{x}(\mathbf{0})\right|$ of the focused $x$-polarized plane wave with the same power. $E_{z}(\mathbf{0})_{\max }$ is for most $N A$ smaller than $\left|E_{x}(\mathbf{0})\right|$ but not much and for $N A \approx n, E_{z}(\mathbf{0})_{\max }$ is even larger than $\left|E_{x}(\mathbf{0})\right|$.

By applying the vector diffraction theory for focusing by a lens of high $N A[1-3,17]$, the electric field $\mathbf{E}^{p}$ in the lens pupil is determined which, according to this theory, yields the field with optimum longitudinal component in the focal plane. The field in a pupil point with polar coordinates $\varrho_{p}$, $\varphi_{p}$, is linked to the field of the plane wave with angles $\vartheta, \varphi$ such that

$$
\varrho_{p}=f \sin \vartheta, \quad \varphi_{p}=\varphi+\pi,
$$

where $f$ is the focal distance. The electric field in the pupil is then [18]:

$$
\begin{aligned}
\mathbf{E}^{p}\left(\varrho_{p}, \varphi_{p}\right) & =\frac{k_{0} n}{2 \pi i} \frac{\sqrt{\cos \vartheta}}{f}\left[A_{\vartheta}(\vartheta, \varphi) \hat{\varrho}+A_{\varphi}(\vartheta, \varphi) \hat{\boldsymbol{\varphi}}\right] \\
& =\frac{i}{2 \pi} \frac{1}{\Lambda} \frac{k_{0} n}{f} \frac{\varrho_{p}}{f^{1 / 2}\left(f^{2}-\varrho_{p}^{2}\right)^{1 / 4}} \hat{\varrho} .
\end{aligned}
$$

The factor $\sqrt{\cos \vartheta}$ guarantees conservation of energy. The optimum pupil field is thus rotational symmetric, linearly polarized in the radial direction and all points of the pupil are in phase. The amplitude is an increasing function of the radial coordinate $\varrho_{p} \leq f N A / n$. A snapshot of the electric field in the pupil is shown in Fig. 5 for $N A / n=0.9$. In Fig. 6 the intensity in the pupil (i.e., $\left|E_{\varrho}\right|^{2}$ ) is shown as a function of $\varrho_{p}$ for several values of $N A / n$. The optimum pupil field can be realized by using three spatial light modulators in series.

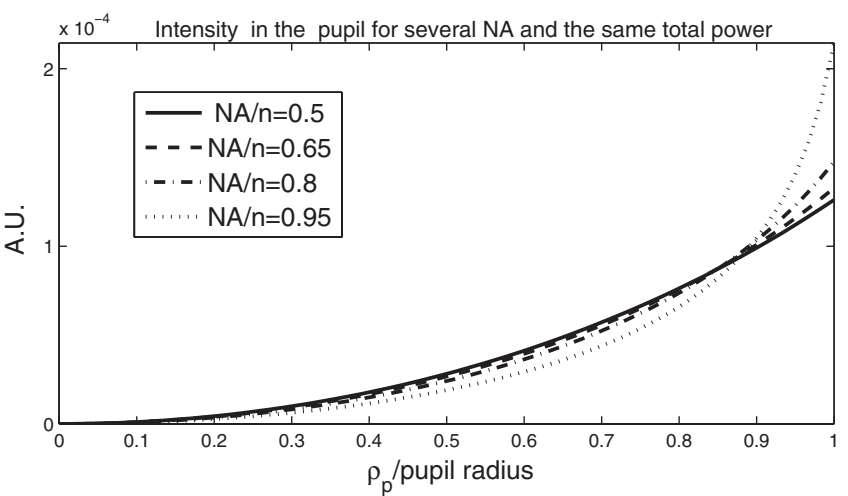

FIG. 6. Cross section of the intensity in the pupil as a function of the radial coordinate, for several values of $N A / n$.

In conclusion, we presented closed formulas for the pupil field which, for given power, yields the largest possible longitudinal electric field component in the focal point of the lens. The pupil field is radially polarized and the electric amplitudes increases with distance to the optical axis. Depending on the numerical aperture, the distribution of the longitudinal component is 15\%-30\% narrower than the classical Airy pattern. In combination with a polarization sensitive resist, the new longitudinal spot thus yields considerable enhancement of resolution.

*h.p.urbach@tudelft.nl

[1] V.S. Ignatowsky, Trans. Opt. Inst. Petrograd, I, papers IV and V (1919).

[2] E. Wolf, Proc. R. Soc. A 253, 349 (1959).

[3] B. Richards and E. Wolf, Proc. R. Soc. A 253, 358 (1959).

[4] S. Quabis, R. Dorn, M. Eberler, O. Glöckl, and G. Leuchs, Opt. Commun. 179, 1 (2000).

[5] S. Quabis, R. Dorn, M. Eberler, O. Glöckl, and G. Leuchs, Appl. Phys. B 72, 109 (2001).

[6] R. Dorn, S. Quabis, and G. Leuchs, Phys. Rev. Lett. 91, 233901 (2003).

[7] C. J. R. Sheppard and A. Choudhurry, Appl. Opt. 43, 4322 (2004).

[8] C. Sanchez (University of Zaragoza), patent application.

[9] L. Novotny, M. R. Beverluis, K. S. Youngworth, and T. G. Brown, Phys. Rev. Lett. 86, 5251 (2001).

[10] X. S. Xie and R. C. Dunn, Science 265, 361 (1994).

[11] Q. W. Zhan, Opt. Express 12, 3377 (2004).

[12] L. E. Helseth, Opt. Commun. 212, 343 (2002).

[13] N. Sanner, N. Huot, E. Audouard, C. Larat, J.-P. Huignard, and B. Loiseaux, Opt. Lett. 30, 1479 (2005).

[14] B. M. I. van der Zande, J. Lub, H. J. Verhoef, W. P. M. Nijssen, and S. A. Lakehal, Liq. Cryst. 33, 723 (2006).

[15] M. Stalder and M. Schadt, Opt. Lett. 21, 1948 (1996).

[16] I. Iglesias and B. Vohnsen, Opt. Commun. 271, 40 (2007).

[17] A. S. van de Nes, L. Billy, S. F. Pereira, and J. J. M. Braat, Opt. Express 12, 1281 (2004).

[18] H.P. Urbach and S. F. Pereira (to be published). 